\title{
Análisis de un brote de meningitis viral en la provincia de Tucumán, Argentina
}

\author{
María Cecilia Freire, ${ }^{1}$ Daniel Marcelo Cisterna, ${ }^{1}$ Karina Rivero, ${ }^{1}$ \\ Gustavo Fabián Palacios, ${ }^{1}$ Inmaculada Casas, ${ }^{2}$ Antonio Tenorio ${ }^{2}$ \\ y Jorge Alberto Gómez ${ }^{3}$
}

RESUMEN Objetivo. Confirmar la existencia de un brote de meningitis viral en 1996 en la provincia de Tucumán, Argentina, y estudiar sus características epidemiológicas.

Métodos. Se analizó información obtenida del Sistema Nacional de Vigilancia Epidemiológica (SINAVE) del Ministerio de Salud de Argentina para el período de 1994-1998, la cual fue provista por la Dirección de Epidemiología de dicho ministerio. Para el cálculo de incidencias se usaron estimaciones poblacionales para los años 1994-1998 realizadas por el Instituto Nacional de Estadística y Censos (INDEC) sobre la base del censo de 1991.

El estudio de frecuencias se realizó mediante el análisis de tablas de contingencia de doble entrada, según el método de ji cuadrado con la corrección de Yates. Se consideró significativo el resultado cuando $\mathrm{P}<0,05$.

Resultados. Se confirmó la presencia de un brote de 189 casos entre el 11 de febrero y el 18 de mayo de 1996. La incidencia de casos en la provincia mostró un aumento entre 1995 y 1996 (de 0,5 a 19,3 casos por 100000 años-persona) y dicha incidencia fue significativamente mayor que la observada en el resto del país (19,3 frente a 2,8 casos por 100000 años-persona). El 75,1\% de los casos ocurrió en niños menores de 9 años (142/189). Se detectó la presencia de Enterovirus (EV) en 65 de las 111 muestras estudiadas (58,6\%). Mediante la reacción en cadena de la polimerasa (RCP) anidada con transcripción inversa se logró detectar EV en $66,3 \%$ (53/80) de los casos estudiados por este método, en comparación con solo 29,6\% (24/81) de los estudiados mediante aislamiento viral. Se identificó echovirus tipo 4 en 15 (68\%) en las 22 muestras tipificadas (5 por aislamiento, 3 por secuenciación y 7 por ambos métodos). Este brote demuestra la capacidad de los EV para diseminarse y producir enfermedad en la población. Durante el brote, por lo menos $56 \%$ de los casos fueron hospitalizados.

Conclusiones. El uso de métodos moleculares permitió el diagnóstico rápido del virus etiológico y posibilitó un mejor control del brote. El reconocimiento temprano de este podría haber evitado la mayoría de las hospitalizaciones y el uso indiscriminado de antibióticos.

Palabras clave Meningitis, brote, Enterovirus, Argentina.

1 Servicio de Neurovirosis, Instituto Nacional de Enfermedades Infecciosas, Administración Nacional de Laboratorios e Institutos de Salud "Dr. Carlos G. Malbrán", Ministerio de Salud, Argentina. Toda la correspondencia debe dirigirse a M.C. Freire a la siguiente dirección postal: Servi-cio de Neurovirosis, Instituto Nacional de Enfermedades Infecciosas, ANLIS “Dr. Carlos G.
Malbrán", Av. Vélez Sársfield 563, Buenos Aires 1281, Argentina. Telefax: (54-11) 4302-5064/(54-11) 4301- 7428. E-mail: mcfreire@anlis.gov.ar

2 Servicio de Microbiología Diagnóstica, Centro Nacional de Microbiología, Instituto de Salud Carlos III, Majadahonda, Madrid, España.

3 Laboratorio de Gastroenteritis Virales, Instituto Nacional de Enfermedades Infecciosas, ANLIS "Dr. Carlos G. Malbrán", Ministerio de Salud, Argentina.
La meningitis, enfermedad que con frecuencia constituye una emergencia médica, se caracteriza por cefalea y fiebre seguidas de confusión e incluso coma (1). Se clasifica en meningitis aséptica (generalmente viral) o purulenta 
(generalmente bacteriana), según el número de leucocitos en el líquido cefalorraquídeo (LCR) (1). Se ha descrito que $58 \%$ de los casos de meningitis son virales (1).

Los EV son la causa más común de meningitis aséptica (2), tanto en casos esporádicos como en brotes epidémicos, y se estima que representan $80 \%$ de los casos de causa conocida (3). En los países de clima templado, las infecciones por EV ocurren fundamentalmente durante el final del verano y el inicio del otoño (4). El género Enterovirus, que es el más extenso de la familia Picornaviridae, abarca a las especies poliovirus (3 serotipos), coxsackievirus A (23 serotipos), coxsackievirus B (6 serotipos), echovirus (26 serotipos) y los nuevos enterovirus (5 serotipos) (5). Los serotipos que más frecuentemente producen meningitis son coxsackievirus B5 y echovirus tipos 4, 6, 9, 11 y 30 (6).

Si bien los brotes de meningitis por EV no se asocian con secuelas (excepto en pacientes inmunodeprimidos) y causan enfermedades de corta duración, afectan a un elevado número de personas $\mathrm{y}$ producen grandes pérdidas económicas (7). En la Argentina, el Sistema Nacional de Vigilancia Epidemiológica (SINAVE) del Ministerio de Salud, que recibe notificaciones de meningoencefalitis desde 1970, recibió 350 informes de casos de meningitis virales en el año 2000. En el período 1995-1998, el Servicio de Neurovirosis del Instituto Nacional de Enfermedades Infecciosas (INEI) identificó EV en 7 de los 8 casos de presuntos brotes de meningitis de los que recibió muestras (8-10). Sin embargo, el estudio de EV - y más aun el estudio de brotes- es excepcional en el país, lo que hace que nuestra comunidad médicosanitaria desconozca la importancia real de los EV como productores de brotes de meningitis. Esta situación dificulta la aplicación de acciones destinadas a lograr un mejor control de los casos durante los brotes y a prevenir la aparición de nuevos casos.

En este trabajo se presentan las estadísticas de salud y los resultados de laboratorio obtenidos a partir de la denuncia de un brote de meningitis que se presentó a principios de 1996 en la provincia de Tucumán, Argentina, y ofrece las principales conclusiones que surgieron como resultado de la evaluación de dicho brote.

\section{MATERIALES Y MÉTODOS}

A fin de determinar si se produjo un brote de meningitis viral y determinar sus características epidemiológicas, se analizó información obtenida del Sistema Nacional de Vigilancia Epidemiológica (SINAVE) para el período de 1994-1998, la cual fue proporcionada por la Dirección de Epidemiología del Ministerio de Salud. Para calcular las incidencias se usaron estimaciones poblacionales para los años 1994-1998 realizadas por el Instituto Nacional de Estadística y Censos (INDEC) sobre la base del censo de 1991 (11).

\section{Antecedentes y envío de las muestras}

Debido a un aumento de casos de meningitis en el Hospital Pediátrico del Niño Jesús, en San Miguel de Tucumán, Argentina, a principios de 1996, el laboratorio de bacteriología del hospital solicitó al Servicio de Neurovirosis del Departamento de Virología del Instituto Nacional de Enfermedades Infecciosas y Administración Nacional de Laboratorios e Institutos de Salud (INEI-ANLIS) "Dr. Carlos G. Malbrán" que estudiara su posible origen viral, según las características clínicas y fisicoquímicas del LCR. En marzo de 1996 se enviaron por vía aérea dos remesas refrigeradas con un total de 125 muestras correspondientes a 106 pacientes internados en el hospital: 96 de LCR, 8 de materia fecal (MF), 10 hisopados faríngeos (HF) y 11 sueros. Todas ellas fueron tomadas en los primeros 10 días del cuadro clínico. Los HF se enviaron embebidos en medio de transporte para virus, y el resto de las muestras en recipientes estériles sin medio de transporte. Cada muestra estaba acompañada de una ficha con los datos del paciente. Desde su llegada al laboratorio y hasta su procesamiento, todas las muestras fueron conservadas a $-70^{\circ} \mathrm{C}$.

\section{Identificación viral}

La detección viral se realizó mediante metodologías clásicas $(12,13)$ y de biología molecular (14, 15). El aislamiento viral se realizó mediante la inoculación de las muestras en células Hep2, Rd y fibroblastos humanos, que permiten detectar los virus que con más frecuencia se asocian con la meningitis. Las muestras fueron tratadas con una mezcla de antibióticos y antimicóticos y después de la inoculación se incubaron durante 7 días a $37^{\circ} \mathrm{C}$ con atmósfera de $5 \%$ de $\mathrm{CO}_{2}$. Si al final del período de incubación no se observó ningún efecto citopático se realizó un nuevo pasaje a ciegas. Los tubos que presentaron algún efecto citopático fueron conservados a $-70{ }^{\circ} \mathrm{C}$ hasta su posterior identificación. Trece de las 14 muestras con efecto citopático característico de EV fueron posteriormente identificadas mediante microneutralización en placas de 96 pocillos (Nunclon ${ }^{\mathrm{TM}}$, Roskilde, Dinamarca) con el Pool de Lim BenyeshMelnick (A-H) provisto por el doctor C. H. Mordhorst, del Statens Seruminstitut, Copenhague, Dinamarca, Centro Colaborador de la Organización Mundial de la Salud para el Plan de Erradicación del Poliovirus Salvaje.

También se realizó la detección de EV en LCR mediante RCP anidada con transcripción inversa de la región 5' no codificante del genoma viral (15), que permite determinar la presencia de cualquiera de los 64 serotipos de EV directamente en el LCR. La extracción del ácido nucleico viral de las muestras clínicas y de los virus aislados se realizó mediante un protocolo previamente descrito (16).

La identificación molecular de los serotipos de EV detectados por RCP y cultivo se realizó mediante secuenciación génica (17). Brevemente, se amplificó la región terminal 3' de la proteína VP1 mediante RCP anidada con transcripción inversa. El producto de la amplificación se secuenció en am-bos sentidos con el sistema comercial Big Dye Terminator Cycle Sequencing Ready Reaction Kit (Applied Biosystems Inc., Foster City, California, EE.UU.). Las secuencias obtenidas se alinearon con el programa MegAlign 
(DNAStar Inc., Madison, Wisconsin, EE.UU.). El serotipo se determinó mediante la comparación de la secuencia parcial de la VP1 del virus aislado con la base de datos del Gene Bank, con el programa CLUSTAL X (versión 1.8). Se consideró que un virus aislado era de un determinado serotipo cuando su secuencia nucleotídica coincidía en por lo menos $75 \%$ con la de un EV prototípico (14).

\section{Datos estadísticos del brote}

La meningitis es una enfermedad que desde 1970 debe ser notificada obligatoriamente al SINAVE no más de 24 horas después de presentarse el caso sospechoso. Dicha notificación debe ser realizada por el médico que atiende el caso. Los casos se clasifican como meningitis virales si el recuento celular del LCR es menor de 1000 células $/ \mathrm{mm}^{3}$.

\section{Análisis estadístico}

El estudio de frecuencias se realizó mediante el análisis de tablas de contingencia de doble entrada, según el método de ji cuadrado con la corrección de Yates. Se consideró el resultado significativo cuando $P<0,05$.

\section{RESULTADOS}

Como se observa en el cuadro 1, la provincia de Tucumán presentó un aumento estadísticamente significativo de la incidencia de meningitis viral en 1996 en relación con 1995 (19,3 frente a 0,5 casos $/ 100000$ años-persona; $P<$ 0,0001 ). Aunque en los dos años siguientes esta incidencia se mantuvo alta en comparación con la de años anteriores a 1996, el número de casos estuvo muy por debajo de los notificados ese año. La incidencia de meningitis viral en 1996 en la provincia de Tucumán fue también significativamente mayor que en el resto del país (19,3 frente a 2,8 casos /100 000 años-persona; $P<0,0001$ ). El número total de casos en Tucumán en 1996 representó casi la tercera parte de los casos del país y ninguna de las provincias limítrofes presentó tasas de la enfermedad superiores a las observadas en el resto del país.

El aumento del número de casos comenzó la semana 6 (del 11 de febrero) y continuó hasta la semana 19 (del 18 de mayo) (figura 1). El aumento observado en este período no se repite en ninguno de los otros años analizados, lo cual confirma que se trata de un brote.

El análisis de los datos por grupo de edad (cuadro 2) permitió determinar que $75,1 \%$ de los casos se observaron en menores de 9 años (142/189). El análisis estadístico no reveló diferencias significativas en la incidencia de casos entre los niños menores de 1 año y los de $1-4$ años (296 frente a 168 casos/100 000 años-niño; $P=0,07)$, ni entre estos últimos y los de 5-9 años (168 frente a 240 casos/100 000 años-niño; $P=0,07$ ). Al juntar estos grupos de edad ( $0-9$ años) se obtuvo una incidencia de 215 casos/100 000 años-niño, la cual fue significativamente superior a la incidencia en el grupo de 10-14 años (108 casos /100 000 años-niño; $P=0,0003$ ). A su vez, la incidencia en este grupo (10-14 años) fue significativamente mayor que la observada en el grupo de mayores de 15 años de edad (6 casos por 100000 años-persona; $P<0,0001)$. Finalmente, no se observaron diferencias significativas entre la incidencia en el grupo de 15-49 años y en el grupo de mayores de 50 años ( 8 frente a 4 casos /100 000 años-persona; $P=0,3$ ). Los datos analizados demuestran que los habitantes de la provincia de Tucumán en su conjunto padecieron 30 veces más casos de meningitis viral que el resto del país (RR: 30; IC 95\%: 24,1 a 37,4) (cuadro 2).

Las edades de los pacientes estudiados en el laboratorio oscilaron entre menos de 1 año y 15 años, con una media de 4 años. De los 103 pacientes sobre cuyo sexo se disponía de información, $43(42 \%)$ eran niñas y 60 $(58 \%)$ eran niños. No se encontraron diferencias significativas $(P=0,0952)$ entre la distribución de edades de los casos notificados al SINAVE y los casos recibidos en el laboratorio (datos no mostrados).

El análisis fisicoquímico de las muestras de LCR mostró entre 7 y 3000 células $/ \mathrm{mm}^{3}$ (media: 261 células $/ \mathrm{mm}^{3}$; DE: 468 células $/ \mathrm{mm}^{3}$ ). El $78 \%$ de las muestras de LCR tenían predominio linfocitario y solo $15 \%$ mostraban predominio de células polimorfonucleares. Estas últimas presentaban en general los mayores conteos $(6,5 \%$ más de 1000 células $/ \mathrm{mm}^{3}$ ). Las concentraciones de glucosa oscilaron entre 0,21 y $0,88 \mathrm{~g} / \mathrm{L}$ (media: 0,51 g/L; DE: 0,11). No se encontró relación entre los parámetros analizados del LCR y la edad de los pacientes, lo que coincide con otros informes (7). No se contó con datos de proteínas y los estudios bacteriológicos fueron todos negativos.

En cultivo, 22,5\% de las muestras de LCR estudiadas (14/62), 87,5\% (7/8) de las de MF y 30,0\% (3/10) de los HF mostraron efectos citopáticos compatibles con EV (cuadro 3). Posteriormente, 13 de las 14 muestras positivas fueron analizadas por microneutralización, y de ellas 12 resultaron ser echovirus tipo 4 (8 LCR, 4 MF) y 1 echovirus tipo 19 (LCR). En cambio, la RCP anidada con

CUADRO 1. Número de casos e incidencia de meningitis viral por año. Provincia de Tucumán, Argentina, 1994-1998

\begin{tabular}{|c|c|c|c|c|c|c|c|c|c|c|c|c|}
\hline & \multicolumn{2}{|c|}{1994} & \multicolumn{2}{|c|}{1995} & \multicolumn{2}{|c|}{1996} & \multicolumn{2}{|c|}{1997} & \multicolumn{2}{|c|}{1998} & \multicolumn{2}{|c|}{ Promedio } \\
\hline & No. & $\rho^{a}$ & No. & I & No. & I & No. & I & No. & I & No. & I \\
\hline Argentina & 798 & 2,3 & 785 & 2,3 & 975 & 2,8 & 955 & 2,7 & 1135 & 3,2 & 929 & 2,7 \\
\hline Tucumán & 1 & 0,1 & 6 & 0,5 & 238 & 19,3 & 74 & 5,9 & 62 & 5,1 & 76 & 6,1 \\
\hline
\end{tabular}

a I: Incidencia o número de casos cada 100000 años-persona. 


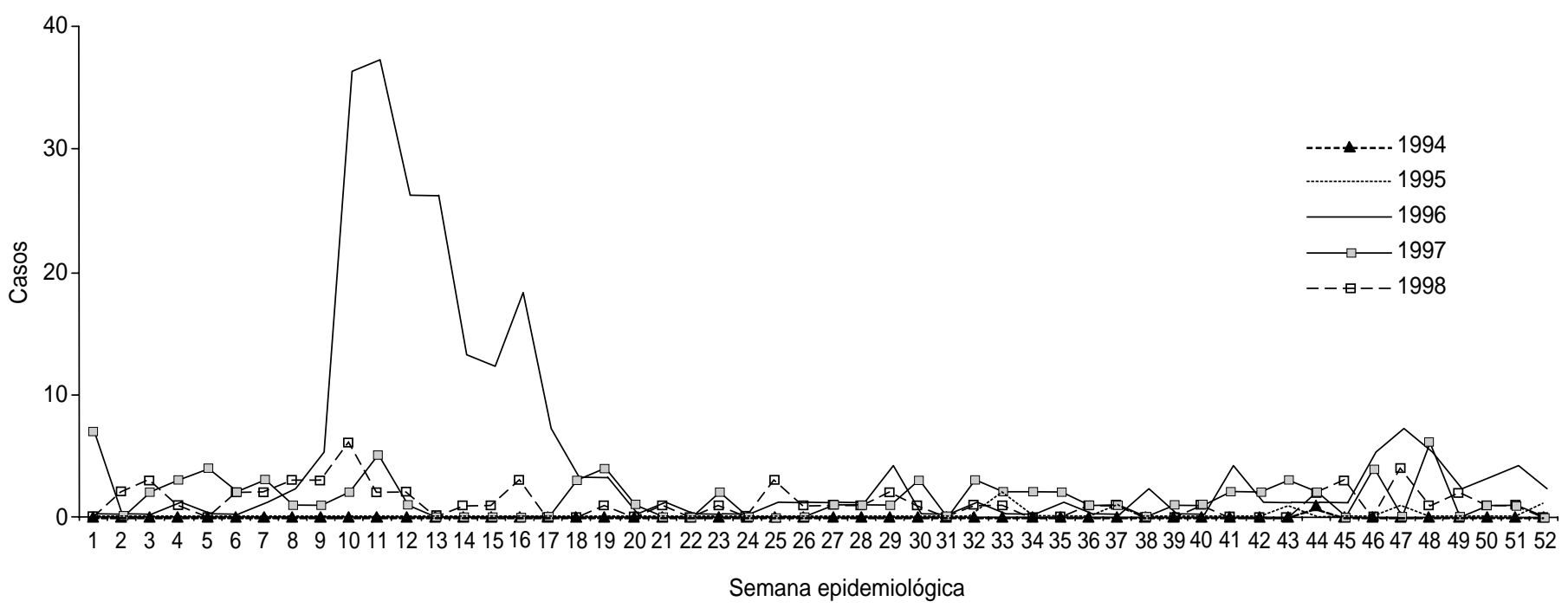

transcripción inversa permitió detectar EV en $62,3 \% \quad(38 / 61)$ de los LCR, en $100 \%$ de las MF y de los $\mathrm{HF}(7 / 7$ y $1 / 1$, respectivamente), y en $63,6 \%$ (7/11) de los sueros (cuadro 3). En general, la técnica de RCP anidada con transcripción inversa detectó $\mathrm{EV}$ en $66,3 \%$ de todas las muestras (53/80), mientras que la técnica de aislamiento viral tuvo éxito solo en $29,6 \%$ de ellas (24/81). Considerando ambas técnicas en su conjunto se detectaron 65 muestras positivas de las 111 estudiadas.

Por último, 16 muestras fueron parcialmente secuenciadas en la región que codifica la proteína VP1 (cuadro 4). El análisis filogenético permitió identificar 7 muestras de las que resultaron positivas para EV tanto por RCP anidada con transcripción inversa como por

CUADRO 2. Número de casos e incidencia de meningitis viral por grupo de edad entre las semanas epidemiológicas 6 y 19 de 1996. Provincia de Tucumán, Argentina

\begin{tabular}{|c|c|c|c|c|c|c|}
\hline \multirow{2}{*}{$\begin{array}{l}\text { Grupo de edad } \\
\text { (en años) }\end{array}$} & \multicolumn{2}{|c|}{ Tucumán } & \multicolumn{2}{|c|}{ Resto de Argentina } & \multirow[b]{2}{*}{ Riesgo relativo } & \multirow[b]{2}{*}{ IC95\% ${ }^{b}$} \\
\hline & No. & $\overline{f^{a}}$ & No. & I & & \\
\hline$<1$ & 17 & 296 & 13 & 8 & 37 & $18,0-76,1$ \\
\hline $1-4$ & 47 & 168 & 28 & 4 & 42 & $26,3-67,1$ \\
\hline $5-9$ & 78 & 240 & 55 & 8 & 30 & $21,3-42,4$ \\
\hline $10-14$ & 35 & 108 & 24 & 4 & 27 & $16,1-45,4$ \\
\hline $15-49$ & 11 & 8 & 18 & 0,4 & 20 & $9,5-42,3$ \\
\hline$\geq 50$ & 1 & 4 & 1 & 0,05 & 80 & $5,0-1279,0$ \\
\hline Total & 189 & 60 & 137 & 2 & 30 & $24,1-37,4$ \\
\hline
\end{tabular}

\footnotetext{
a I: Incidencia o número de casos cada 100000 años-persona.
}

b IC95\%: intervalo de confianza de $95 \%$. aislamiento viral como echovirus tipo 4, mientras que de otras 9 muestras positivas solo por RCP anidada con transcripción inversa se encontró echovirus tipo 4 en otras 3 muestras (MF), en 3 echovirus tipo 7 ( 2 LCR, $1 \mathrm{MF}$ ), en 2 echovirus tipo 9 (LCR) y en 1 echovirus tipo 29 (MF). De las 15 muestras con echovirus tipo 4 identificados, en 7 se llegó al mismo resultado por ambos métodos, en 3 solo por secuenciación y en 5 solo por aislamiento.

\section{DISCUSIÓN}

Los resultados presentados demuestran la existencia de un brote de meningitis por EV en la provincia de Tucumán, Argentina, entre las semanas
6 y 19 de 1996, que implicó al menos a 189 pacientes. Este brote permitió analizar los parámetros epidemiológicos y de laboratorio de meningitis por EV en una población determinada y por primera vez se describen las características de un brote de este tipo en la Argentina.

La incidencia media de meningitis viral en la Argentina entre 1994 y 1998 fue de 2,7 casos/100 000 años-persona (recorrido: 2,3-3,2). En cambio, durante las semanas del brote en la provincia de Tucumán, la incidencia fue 30 veces mayor que en el resto del país (60 frente a 2 casos/100 000 años-persona, respectivamente). En los años subsiguientes la incidencia de casos en la provincia mantuvo niveles mayores a los observados antes del brote, probablemente debido a una mejora en la notificación como consecuencia del mismo. Los primeros casos se observaron en febrero y los últimos en mayo, con un pico en el mes de marzo. Esta distribución coincide con trabajos previos que describen una mayor circulación de EV durante el verano y el otoño (4). El origen del brote permanece desconocido; sin embargo, el análisis de la curva epidemiológica parece indicar que la diseminación posterior fue por mecanismos de transmisión de persona a persona. El grupo de edad más comprometido fue el de 0 a 9 años (75\% de los casos), y la incidencia en este 
CUADRO 3. Número de muestras recibidas, estudiadas y positivas según el método de estudio utilizado. Provincia de Tucumán, Argentina, 1996

\begin{tabular}{|c|c|c|c|c|c|c|}
\hline \multirow[b]{2}{*}{ Método } & \multicolumn{2}{|c|}{ Cultivo } & \multicolumn{2}{|c|}{$\mathrm{RCP}$} & \multicolumn{2}{|c|}{ Cultivo o RCP } \\
\hline & Estudiadas/Total & Positivas, \% & Estudiadas/Total & Positivas, \% & Estudiadas/Total & Positivas, \% \\
\hline LCR & $62 / 96$ & $14(22,5)$ & $61 / 96$ & $38(62,3)$ & $82 / 96$ & $47(57,1)$ \\
\hline $\mathrm{HF}$ & $10 / 10$ & $3(30,0)$ & $1 / 9$ & $1(100,0)$ & $10 / 10$ & $4(40,0)$ \\
\hline Suero & $1 / 11$ & $0(0,0)$ & $11 / 11$ & $7(63,6)$ & $11 / 11$ & $7(63,6)$ \\
\hline Total & $81 / 125$ & $24(29,6)$ & $80 / 125$ & $53(66,3)$ & $111 / 125$ & $65(57,0)$ \\
\hline
\end{tabular}

Nota: LCR: líquido cefalorraquídeo; MF: materia fecal; HF: hisopado faríngeo; RCP: reacción en cadena de la polimerasa.

grupo fue el doble de la observada en el grupo de 10 a 14 años y casi 36 veces mayor que la observada en los mayores de 15 años. Este hecho podría indicar que los adultos se encontraban protegidos debido a reiteradas infecciones por EV durante su infancia (18). El gran número de personas susceptibles, así como el comportamiento común en la infancia, pueden haber favorecido la eficiente transmisión de los EV en este grupo de edad.

No se encontró relación entre los parámetros bioquímicos de las 96 muestras de LCR recibidas y la edad de los pacientes (7). El diagnóstico por RCP anidada con transcripción inversa fue más sensible que el de aislamiento viral $(66,3 \%$ frente a 29,6\%), mientras que la combinación de ambos métodos permitió identificar EV en 65 de las 111 muestras estudiadas.

Por otra parte, los resultados obtenidos indican que los EV se recuperaron con mayor eficiencia en las heces $(87,5 \%$ de muestras positivas). Los estudios de serotipificación por métodos clásicos y de biología molecular señalan al echovirus tipo 4 como el agente más probable del brote, ya que en $68 \%(15 / 22)$ de las muestras tipificadas por cualquiera de dichos métodos identificaron este patógeno ( 5 por aislamiento, 3 por secuenciación y 7 por ambos). Esta es la primera vez que se detecta un brote de meningitis por echovirus tipo 4 en la Argentina, ya que los brotes analizados entre 1995 y 1998 fueron asociados a echovirus tipo 9 y echovirus tipo 30. Adicionalmente, en el brote de Tucumán se detectó también la circulación de echooirus tipos 7, 9, 19 y 29.

Este brote confirma la capacidad de los EV para diseminarse y producir

CUADRO 4. Serotipos de Enterovirus tipificados según el método de identificación empleado. Provincia de Tucumán, Argentina, 1996

\begin{tabular}{lcc}
\hline Serotipo & Cultivo/Neutralización & RCPa/Secuenciación (VP1) \\
\hline E4 & 12 & 10 \\
E7 & 0 & 3 \\
E9 & 0 & 2 \\
E19 & 1 & 0 \\
E29 & 0 & 1 \\
Sin tipificar & 0 & 0 \\
$\quad$ Total & 13 & 16 \\
\hline
\end{tabular}

a Reacción en cadena de la polimerasa.

enfermedad en la población. De acuerdo con los datos analizados, ocurrieron 189 casos y se hospitalizaron al menos 106 pacientes ( $56 \%$ de los casos). La carencia de estudios de brotes y las dificultades que presenta el diagnóstico de los EV entorpecen el control correcto de situaciones de este tipo. El uso de métodos moleculares en los hospitales permite el diagnóstico rápido del virus y posibilita controlar los brotes de manera más adecuada. Por otra parte, el reconocimiento temprano del mismo podría haber evitado la mayoría de las hospitalizaciones y el uso indiscriminado de antibióticos.

Uno de los componentes más importantes del sistema de salud pública ante un brote de EV para interrumpir su transmisión es la educación, con énfasis en la higiene personal (lavado de manos, uso individual de los utensilios de comida y uso personal, etc.). Ante un brote de meningitis aséptica, la acción del sistema de salud —basada en la educación sanitaria $y$ en buenas campañas publicitarias - podría reducir el uso de recursos costosos. Los pacientes con meningitis viral no se benefician de la realización de una tomografía com- putadorizada ni del tratamiento antibiótico indiscriminado, y hasta es dudosa la necesidad de hospitalizarlos. Si los parámetros del LCR sugieren una meningitis y existe la sospecha de un brote por EV, los pacientes deberían tratarse solo en consultas ambulatorias, reservándose la hospitalización para aquellos pacientes que presenten manifestaciones atípicas. Las herramientas microbiológicas y epidemiológicas utilizadas en el presente trabajo, más la aplicación sistemática del estudio de brotes, facilitarían este cambio de conducta.

Agradecimiento. Se agradece a la Dra. Ana María Trejo, del Laboratorio de Bacteriología del Hospital del Niño Jesús de Tucumán, Argentina, su colaboración en el envío de las muestras; al Dr. C. H. Mordhorst del Statens Seruminstitut la provisión del Pool de Lim Benyesh-Melnick (A-H); a Flavio Vergara el procesamiento de las muestras, y a la Dra. Mirtha Eimman, de la Dirección de Epidemiología del Ministerio de Salud, la provisión de las estadísticas de salud analizadas. 
1. Tunkel AR, Scheld WM. Acute meningitis. En: Mandell GL, Bennett JE, Dolin R, eds. Principles and practice of infectious diseases. Philadelphia: Churchill Livingstone; 2000: 959-997.

2. Hammer SM, Connolly KJ. Viral aseptic meningitis in the United States: clinical features, viral etiologies, and differential diagnosis. Curr Clin Trop Infect Dis 1992;12:1-25.

3. Sawyer MH, Holland D, Aintablian N, Connor JD, Keyser EF, Waecker NJ, Jr. Diagnosis of enteroviral central nervous system infection by polymerase chain reaction during a large community outbreak. Pediatr Infect Dis J 1994;13(3):177-182.

4. Morens D, Pallansch M. Epidemiology. En: Rotbart H, ed. Human enterovirus infections. Washington, D.C.: ASM Press; 1995. Pp. 3-23.

5. Oberste M, Schnurr D, Maher K, Busaidy-al S, Pallansch M. Molecular identification of new picornaviruses and characterization of a proposed enterovirus 73 serotype. J Gen Virol 2001;82(Pt2):409-416.

6. Centers for Disease Control and Prevention. Enterovirus surveillance. United States, 19971999. MMWR 2000;49(40):913-916.

7. Rice SK, Heinl RE, Thornton LL, Opal SM. Clinical characteristics, management strategies, and cost implications of a statewide outbreak of enterovirus meningitis. Clin Infect Dis 1995;20(4):931-937.
8. Cisterna D, Palacios Poggio G, Rivero K, Girard D, Freire MC. Brotes de meningitis aséptica en la Argentina. VIII Congreso Argentino de Microbiología. Buenos Aires, Argentina. Buenos Aires: AAM; 1998

9. Freire MC, Casas I, Tenorio A, Palacios Poggio G, Cisterna D. Análisis de un brote de meningitis viral en Tucumán. $32^{\circ}$ Congreso Argentino de Pediatría. Salta, Argentina: SAP; 2000.

10. Palacios Poggio G, Cisterna D, Berra A, Freire MC. Outbreak of hemorrhagic conjunctivitis in Argentina in the summer of 1997. Annual Meeting of the Association for Research in Vision and Ophthalmology, Fort Lauderdale, Florida, 1998.

11. Instituto Nacional de Estadística y Censos. Proyecciones de la población por provincia según sexo y grupos de edad, 1990-2010. Buenos Aires: INDEC; 1995.

12. Chonmaitree T, Baldwin CD, Lucía HL. Role of the virology laboratory in the diagnosis and management of patients with central nervous system disease. Clin Microbiol Rev 1989; 2(1):1-14.

13. Wildin S, Chonmaitree T. The importance of the virology laboratory in the diagnosis and management of viral meningitis. Am J Dis Child 1987;141(4):454-457.

14. Palacios G, Casas I, Tenorio A, Freire C. Molecular identification of enterovirus by analyzing a partial VP1 genomic region with different methods. J Clin Microbiol 2002;40(1): 182-192.

15. Palacios Poggio G, Cisterna D, Freire MC, Cello J. RT-nested PCR for the detection of enterovirus in biological samples from patients with suspected enteroviral infections. Rev Argent Microbiol 2000;32(4):165-172.

16. Casas I, Powell L, Klapper PE, Cleator GM. New method for the extraction of viral RNA and DNA from cerebrospinal fluid for use in the polymerase chain reaction assay. J Virol Methods 1995;53(1):25-36.

17. Casas I, Palacios G, Trallero G, Cisterna D, Freire $\mathrm{M}$, Tenorio A. Molecular characterization of human enteroviruses in clinical samples: comparison between VP2, VP1, and RNA polymerase regions using RT-nested PCR assays and direct sequencing of products. J Med Virol 2001;65(1):138-148.

18. Dagan R, Menegus M. Non-polio enteroviruses and the febrile infant. En: Rotbart H, ed. Human enterovirus infections. Washington: ASM Press; 1995:239-254.

Manuscrito recibido el 4 de marzo de 2002 y aceptado, tras revisión, el 15 de noviembre de 2002. the province of Tucuman, Argentina, and to study the outbreak's epidemiological characteristics.

\section{Analysis of an outbreak of viral meningitis in the province of Tucuman, Argentina}

Methods. We analyzed information from the National Epidemiological Surveillance System of the Ministry of Health (MOH) of Argentina for 1994-1998 that had been provided by the $\mathrm{MOH}^{\prime}$ s Bureau of Epidemiology. We calculated incidence rates using population estimates for the years 1994-1998 developed by the National Statistics and Census Institute, based on the 1991 census. We studied frequencies with contingency tables, using the chi-square method with Yates' correction. Results were considered significant when $P<0.05$.

Results. We confirmed the occurrence of an outbreak of 189 cases of viral meningitis between 11 February and 18 May 1996. The incidence of cases in Tucuman province increased between 1995 and 1996, from 0.5 to 19.3 cases per 100000 person-years. That 1996 rate in Tucuman was significantly higher than what was seen in the rest of the country (2.8 cases per 100000 person-years). Of the 189 cases, 142 of them $(75.1 \%)$ occurred in children less than 9 years old. Out of 111 samples studied, 65 of them $(58.6 \%)$ were enterovirus-positive. Through reverse transcription-nested polymerase chain reaction, enteroviruses were found in $66.3 \%(53 / 80)$ of the cases studied by this method, versus in only $29.6 \%(24 / 81)$ of the cases studied through viral isolation. In the 22 samples that were serotyped, echovirus type 4 was identified in 15 of them (68\%): 5 by isolation, 3 by sequencing, and 7 by both methods. During the Tucuman outbreak, at least $56 \%$ of the cases were hospitalized. This viral meningitis outbreak shows the capacity of enteroviruses to spread and cause disease.

Conclusions. The use of molecular methods makes it possible to rapidly diagnose the etiological virus and to better control an outbreak. Recognizing this outbreak in Tucuman sooner could have averted the majority of the hospitalizations and the indiscriminate use of antibiotics. 
El archivo disponible recibió correcciones según ERRATA publicada en el volumen 14 número 1 de la revista. 